\title{
Experience with the sodium sulphate-Alcian Blue stain for amyloid in cardiac pathology
}

\author{
ARIELA POMERANCE, GERARD SLAVIN, AND JOSEPHINE McWATT \\ From the Department of Histopathology, Northwick Park Hospital and Clinical Research Centre, \\ Harrow, Middx.
}

SYNOPSIS The sodium sulphate-Alcian Blue (SAB) method, which stains amyloid green, was evaluated in 220 hearts from elderly patients. The technique proved superior to the Congo red, crystal violet, and thioflavine $\mathrm{T}$ methods used either singly or as a battery for the demonstration of cardiac amyloid. Amyloid was easily identified under the $\times 3$ objective, even in small amounts. A few non-amyloid components stained varying shades of green but were easily distinguished on morphological grounds. No false positive or equivocal reactions occurred, and in particular elastic laminae and paravascular connective tissue were not tinctorially confused with amyloid. The SAB stain is technically simple and consistently reproducible, and no special light source is required for examination. An additional advantage in cardiac pathology is the simultaneous demonstration of any fibrosis, basophilic myofibre degeneration, tissue mast cells and mucoid degeneration of valves present.

Amyloid deposition is a well recognized association of ageing in many animal species, including man. The clinical significance of the relatively small quantities of amyloid present in many organs still remains to be fully evaluated, as does any relationship to immunological alteration in senescence (Walford, 1969; Husby and Natvig, 1974).

As part of a clinicopathological study of heart disease in the elderly, the hearts of all patients coming to necropsy from the geriatric wards were examined for cardiac amyloid using crystal violet, alkaline Congo red, and thioflavine $T$ techniques. This is a time-consuming process for both technician and pathologist and may be impracticable as a routine in many hospitals with high geriatric necropsy rates. Furthermore, each of these methods has particular disadvantages when applied to cardiac pathology. The modified sodium sulphate-Alcian Blue technique recently described by Lendrum et al (1972) for staining amyloid in paraffin sections seemed a promising alternative, and this communication records experience with the method.

\section{Material and Methods}

Two hundred and twenty hearts were examined. The patients had all died in the geriatric wards of this hospital. A minimum of three blocks was

Received for publication 28 April 1975. examined from each heart, one from the atrial septum and one from each ventricle. In many cases additional blocks were taken for other diagnostic purposes and these were also examined. The tissue was fixed in $10 \%$ formol-saline and stained by the following methods:

(a) crystal violet staining and examination for metachromasia;

(b) alkaline Congo red staining with examination for green birefringence by polarized light;

(c) thioflavine $\mathrm{T}$ staining and examination for fluorescence by ultraviolet light.

These three methods are those currently in general use for identification of amyloid (Drury and Wallington, 1967). They are subsequently referred to as the 'standard battery'.

(d) the sodium sulphate-Alcian Blue (SAB) method, with Van Gieson counterstain, as follows:

Take sections to water; wash 10 minutes.

Transfer to acetic-alcohol, one to two minutes.

Stain in $\mathbf{S A B}^{1}$ solution, two hours.

Transfer to acetic-alcohol $(45 \mathrm{ml}$ each of $95 \%$ ethanol and distilled water, with $10 \mathrm{ml}$ glacial acetic acid), one to two minutes.

Wash in water.

1SAB solution: $45 \mathrm{ml} 1 \%$ Alcian Blue $8 \mathrm{GX}$ in $95 \%$ ethanol, $45 \mathrm{ml} 1 \%$ sodium sulphate hydrate in distilled water, and $10 \mathrm{ml}$ glacial acetic acid. This should be freshly prepared and allowed to stand for 30 minutes before use. 


\begin{tabular}{|c|c|c|c|c|c|}
\hline \multirow[t]{2}{*}{ Material } & \multirow[t]{2}{*}{ Grade of Amyloid ${ }^{1}$} & \multicolumn{3}{|c|}{ Staining Reactions } & \multirow{2}{*}{$\frac{.}{S A B}$} \\
\hline & & Crystal Violet & Congo Red & Thioflavine $T$ & \\
\hline \multirow{2}{*}{$\begin{array}{l}\text { Group } 1 \\
110 \text { cases screened with crystal } \\
\text { violet, Congo red, and thioflavine } \\
\text { T methods. } \\
\text { Amyloid diagnosed by two } \\
\text { methods in } 26 \text { cases }\end{array}$} & $\begin{array}{l}4 \& 5 \\
3 \\
2 \\
1\end{array}$ & $\begin{array}{r}10 \\
5 \\
3 \\
0\end{array}$ & $\begin{array}{r}10 \\
5 \\
5 \\
6\end{array}$ & $\begin{array}{l}8 \\
5 \\
5 \\
6\end{array}$ & $\begin{array}{r}10 \\
5 \\
5 \\
6\end{array}$ \\
\hline & $\begin{array}{l}\text { One positive } \\
\text { staining reaction } \\
\text { only }\end{array}$ & 0 & 2 & 1 & 3 \\
\hline \multirow[t]{2}{*}{$\begin{array}{l}\text { Group } 2 \\
36 \text { cases screened with all four } \\
\text { methods. } \\
\text { Amyloid diagnosed by two } \\
\text { methods in } 20 \text { cases }\end{array}$} & $\begin{array}{l}4 \& 5 \\
3 \\
2 \\
1\end{array}$ & $\begin{array}{l}1 \\
2 \\
3 \\
0\end{array}$ & $\begin{array}{r}1 \\
2 \\
3 \\
13\end{array}$ & $\begin{array}{l}1 \\
2 \\
0 \\
3\end{array}$ & $\begin{array}{r}1 \\
2 \\
3 \\
13\end{array}$ \\
\hline & $\begin{array}{l}\text { One positive } \\
\text { staining reaction } \\
\text { only }\end{array}$ & 0 & $\mathbf{0}$ & 0 & 1 \\
\hline $\begin{array}{l}\text { Group } 3 \\
74 \text { cases screened with SAB } \\
\text { method only. } \\
\text { Amyloid diagnosed by two } \\
\text { methods in } 34 \text { cases }\end{array}$ & $\begin{array}{l}4 \& 5 \\
3 \\
2 \\
1 \\
\text { One positive } \\
\text { staining reaction } \\
\text { only }\end{array}$ & $\begin{array}{l}6 \\
3 \\
0 \\
0 \\
0\end{array}$ & $\begin{array}{r}6 \\
3 \\
7 \\
18 \\
0\end{array}$ & \}$_{2}$ Not done & $\begin{array}{r}6 \\
3 \\
7 \\
18 \\
5\end{array}$ \\
\hline
\end{tabular}

Table Positive staining reactions in 220 hearts examined for senile cardiac amyloidosis

${ }^{1}$ Grade 1 early atrial deposits, insufficient for demonstration on crystal violet staining

" 2 atrial deposits only but well marked

" 3 atrial deposits with occasional small foci of ventricular amyloid

" 4\&5 atrial deposits with moderate and marked ventricular involvement

Alkalinize in $80 \%$ ethanol saturated with borax, 30 minutes.

Wash in water.

Stain nuclei in ferrous haematein, five minutes.

Wash in water.

Take each slide individually and differentiate by dipping in $80 \%$ ethanol saturated with picric acid, 20 to 30 seconds; rinse briefly in water to remove the alcohol and stain two to three minutes in picrofuchsin. Flush off the picrofuchsin rapidly with $95 \%$ ethanol and so to absolute ethanol and then to xylene and mount in BPS.

\section{Results}

The material was divided into three groups (table).

GROUP 1

Group 1 consisted of 29 cases in which amyloid had originally been diagnosed by the standard battery of stains. With three exceptions the diagnosis had been made on a positive reaction with at least two of the three methods. The exceptions were a small area of fine fluorescent fibres around myofibres on thioflavine $\mathrm{T}$ staining which was morphologically typical of early senile cardiac amyloidosis but could not be demonstrated by the Congo red method, and two cases with small foci of green birefringence with the Congo red technique but where the intensity of fluorescence with thioflavine $T$ was too poor for convincing diagnosis.

All the amyloid deposits noted by any of the standard methods were stained green by the SAB method. The shade of green varied from rather pale with a greyish tinge to brilliant jade green (figs $1,3,5$ ). There was no difficulty in identifying the amyloid with the lowest power $(\times 3)$ objective. Small quantities were identified far more rapidly and easily than with any of the other techniques. With large deposits the areas of green staining tissue were comparable to the areas of metachromasia seen in sections stained with crystal violet (fig 4). In two cases there were well-marked amyloid deposits which had failed to stain satisfactorily with crystal violet; these were satisfactorily stained with the SAB method. With minor deposits the SAB-stained sections showed a more extensive fine network of amyloid fibres than was apparent with thioflavine $T$ or Congo red.

In addition sections adjacent to examples of 'false positive' reactions from fluorescent endocardial fibrin were examined, as were all doubtful cases where it was not possible to distinguish early vascular or endocardial amyloid from elastic laminae on thioflavine $\mathrm{T}$ staining. In the cases which had shown doubtful or false positive reactions by the 


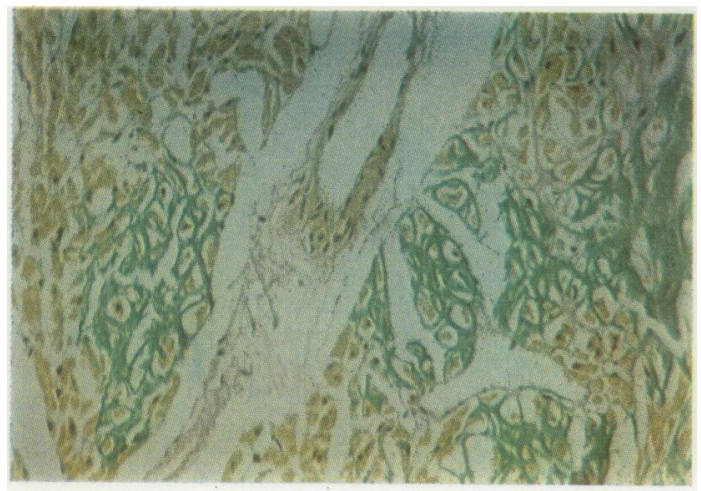

Fig 1

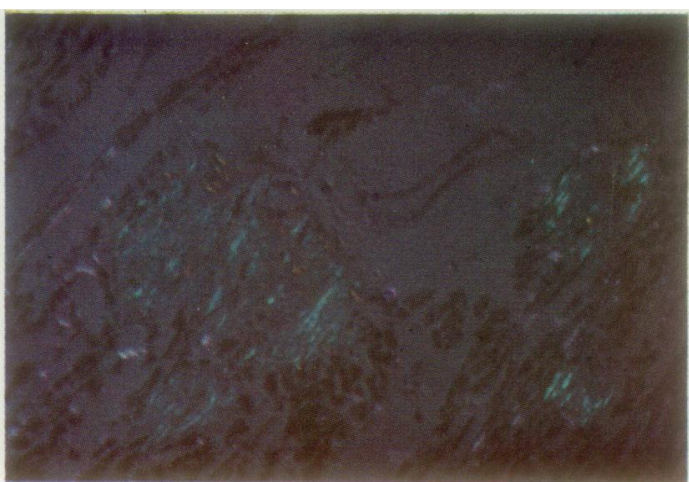

Fig 2

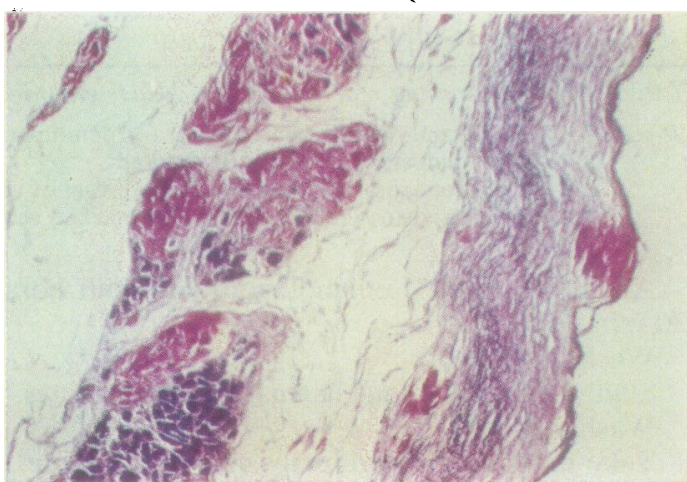

Fig 4

Fig 1 Section of left ventricle stained with $S A B$ technique showing extensive green deposits of amyloid surrounding and compressing muscle fibres ( $\times 280)$.

Fig 2 Adjacent section stained with Congo red and viewed through crossed polars. Green and orange birefringent areas correspond to the green-staining amyloid in fig 1 ( $\times 250)$.

Fig 3 Section of atrial septum showing marked amyloid deposition; for comparison with fig $4(S A B \times 50)$.

Fig 4 Adjacent section stained with crystal violet. The quantity of tissue staining as amyloid appears the same as in fig $3(\times 50)$.

Fig 5 Very early amyloidosis. Green staining material is seen in capillary wall, and very fine deposits are present around some adjacent myofibres. Congo red staining of an adjacent section showed typical green birefringence in the vascular deposits $(S A B \times 800)$.

[Figure 3 from a transparency taken on Kodachrome photomicrography colour film; Figures 1, 2, 4, and 5 from Ektachrome (high-speed) transparencies.] 
thioflavine $\mathbf{T}$ or Congo red techniques, the appearances with the SAB stain were unequivocal. Fibrin and elastic fibres, both of which often show fluorescence in thioflavine $T$ staining, did not show any green colour. Fibrin appeared yellow and elastic fibres were not distinguishable from other endocardial or vascular components. Paravascular connective tissue, which not infrequently shows small green foci by the Congo red method, showed only the red staining of collagen on SAB staining.

Green staining tissues other than amyloid were easily distinguishable on morphological grounds in the SAB-stained section alone: tissue mast cells stained a dense bright green and showed typical cytoplasmic granularity. Red blood cells occasionally appeared very pale green; calcium deposits stained a dirty dark blue/green. Basophilic degeneration of myofibres also appeared as solid pale green areas but these were clearly localized within the myofibres, as was the green granularity produced by lipofuscin in large quantities.

\section{GROUP 2}

Following the initial retrospective study the SAB technique was added to the standard battery already used routinely, and the hearts of all necropsy cases from the geriatric wards were screened using all four methods for a period of six months. In this time 36 hearts were examined and amyloid was demonstrated in 20 cases. All were positive with the SAB stain and by one or more of the other three methods. In no case was amyloid demonstrated by the standard battery and not by the SAB method. In a further case very small green deposits were noted in the SAB-stained sections, mainly in the walls of a few subendocardial atrial capillaries. These did not show Congo red birefringence or convincing thioflavine $T$ fluorescence, but the appearance in the SAB-stained sections was indistinguishable from that in similar vessels in which Congo red birefringence was demonstrable (fig 5).

GROUP 3

In 74 subsequent cases the SAB technique was used as the sole screening method for senile cardiac amyloid. Congo red and/or crystal violet stainingwas used for confirmation of positive results only. Amyloid was recognized in 34 cases. Five cases stained with SAB showed green areas in the walls of occasional atrial capillaries without confirmatory green birefringence in the Congo red technique.

One hundred and ten hearts (groups 2 and 3) have now been screened using the SAB technique either alone or in combination with the 'standard battery'. Amyloid deposits have been recognized in 54 cases. In many the confirmatory green birefringence in the Congo red method was seen only by examining previously marked fields under the $\times \mathbf{4 0}$ objective. Deposits of this small size are easily overlooked without the use of the SAB stain. The $49 \%$ incidence of senile cardiac amyloid found with the SAB technique compares with $26 \%$ in the 110 earlier cases screened by the standard battery of stains for amyloid. The difference was mainly in the ease of recognition of very small atrial deposits which comprised 31 of the cases found by SAB staining; only six such foci were diagnosed in the material screened by thioflavine $T$ and Congo red techniques (group 1).

\section{Discussion}

This study was concerned primarily with the diagnosis of amyloid in the aged heart, where it is commonly present but generally undiagnosed unless special stains are employed. Before using the SAB method 110 hearts had been examined for amyloid by the three most widely accepted techniques for its histological identification-Congo red viewed by polarized light, thioflavine $\mathrm{T}$ with ultraviolet light, and crystal violet. None of these methods is entirely satisfactory, and we have experienced all the problems noted by earlier workers in other sites, who found thioflavine $T$ variable (Cooper, 1969; McKinney and Grubb, 1965) and nonspecific (Burns et al, 1967; Wright et al, 1969; Wolman and Bubis, 1965; Cooper, 1969; Klatskin, 1969; McKinney and Grubb, 1965; Lehner, 1965), crystal violet relatively insensitive (Cooper, 1969) and the green polarization colour with Congo red staining not diagnostic of amyloid (Wright et al, 1969; Klatskin, 1969; Reissenweber and Decaro, 1969; Shapell et al, 1973). In addition, thioflavine $T$ powder deteriorated on storage and showed batch variability.

The present study has shown that the SAB technique is superior to the other three methods, either individually or in combination, for the identification of even small quantities of amyloid in the heart. All tissues showing both the morphological and tinctorial characteristics of amyloid by any of the other three methods showed a green colour in SAB-stained sections which was easily identified with the lowest (x3) objective of an ordinary laboratory microscope. With thioflavine $T$ staining fluorescence was often observed in fibrin, calcium deposits, and elastic laminae, but none of these substances caused diagnostic confusion in SAB-stained sections. The Congo red staining often showed green birefringence in paravascular and interstitial connective tissue but in SAB-stained sections these areas stained as fibrous tissue only. Crystal violet failed to show metachromasia in three hearts with extensive foci of 
amyloid visible on Congo red or thioflavine $T$ staining. No such 'false negatives' occurred with the SAB method.

Although substances other than amyloid in the heart also stained green their morphological and anatomical relationships were clearly apparent in the SAB-stained section. Tissue mast cells were identifiable by their granularity; the green of calcification was heavily tinged with dark blue; basophilic myofibre degeneration and lipochrome pigment stained grey-green but were clearly within the myofibre cytoplasm. Early myofibre degeneration may show a green tinge but the cytoplasmic vacuolation and granularity were obvious in the SAB stain. Mucoid degeneration of valve fibrosa stains blue/ green with the SAB stain but has no morphological resemblance to the solid pale green nodules of valvular amyloid.

Since adopting the SAB stain as the routine screening method the proportion of hearts in which amyloid was detected has increased from $26 \%$ to $49 \%$, mainly due to recognition of very early atrial deposits. Although easily seen in SAB-stained sections, confirmation by other techniques of these deposits was often difficult, requiring careful scrutiny and rotation of previously marked areas under $\times 20$ or $\times 40$ objectives before the characteristic green polarization colour on Congo red staining was identified. Clearly, most such deposits would have been overlooked if only the standard battery had been used. While these deposits may be of little individual clinical significance their identification may be important in more fundamental studies on the ageing process.

In four cases small green foci morphologically typical of amyloid were seen only in very occasional capillaries. No Congo red birefringence or convincing thioflavine $T$ fluorescence could be seen. However, similar slightly more extensive lesions did show typical Congo red birefringence, and we believe that these are small foci of cardiac amyloidosis but were lacking an area of suitable orientated amyloid fibrils in the plane of the section to produce positive Congo red staining and green birefringence (Wolman and Bubis, 1965).

In this study the SAB technique has proved to be more sensitive than the standard battery of stains either singly or in combination in the detection of cardiac amyloid. In addition the method has a number of other practical advantages in cardiac pathology. It is technically simple, no variability or deterioration of stain was encountered, and satis- factory results were consistently obtained. For both diagnostic and research purposes it offers rapid and easy screening for amyloid without the need for examination of multiple stains or for the comparatively sophisticated microscopes necessary to provide high intensity and ultraviolet lighting or strainfree objectives. Finally, the same SAB-stained sections provide additional useful data such as the extent of myocardial fibrosis, the presence of excessive basophilic myofibre degeneration, the number and distribution of tissue mast cells, and the presence and severity of mucoid degeneration of valves.

We are grateful to $\mathrm{Mr}$ J. Clark and Dr M. Bennett for assistance with the colour photography, and to the British Heart Foundation for most of the cost of the colour plate.

\section{References}

Burns, J., Pennock, C. A., and Stoward, P. J. (1967). The specificity of the staining of amyloid deposits with thioflavine T. J. Path. Bact., 94, 337-344.

Cooper, J. H. (1969). An evaluation of current methods for the diagnostic histochemistry of amyloid. J. clin. Path. 22, 410-413.

Drury, R. A. B. and Wallington, E. A., eds. (1967). Carleton's Histological Technique. Oxford University Press, London.

Husby, G. and Natvig, J. B. (1974). A serum component related to non-immunoglobulin amyloid protein AS, a possible precursor of the fibrils. J. clin. Invest., 53, 1054-1061.

Klatskin, G. (1969). Nonspecific green birefringence in Congo Red-stained tissues. Amer. J. Path., 56, 1-13.

Lehner, T. (1965). Juxta glomerular apparatus staining with thioflavine-T fluorochrome, and its confusion with amyloid. (Letter) Nature (Lond.), 206, 738.

Lendrum, A. C., Slidders, W. and Fraser, D. S. (1972). Renal hyaline: A study of amyloidosis and diabetic fibrinous vasculosis with new staining methods. J. clin. Path., 25, 373-396.

McKinney, B. and Grubb, C. (1965). Non-specificity of thioflavine-T as an amyloid stain. Nature (Lond.), 205, 1023-1024.

Reissenweber, N. J., and Decaro, J. (1969). Dichroism with Congo Red. A specific test for amyloid? Virchows Arch. path. Anat., 347, 254-259.

Shappell, S. D., Marshall, C. E., Brown, R. E., and Bruce, T. A. (1973). Sudden death and the familial occurrence of mid-systolic click, late systolic murmur syndrome. Ciculation, 48, 1128-1134.

Walford, R. L. (1969). The Immunologic Theory of Aging. Munksgaard, Copenhagen.

Wolman, M. and Bubis, J. J. (1965). The cause of the green polarization colour of amyloid stained with Congo Red. Histochemie, 4, 351-356.

Wright, J. R., Calkins, E., Breen, W. J., Stolte, G. and Schultz, R. T. (1969). Relationship of amyloid to aging Medicine (Baltimore), 48, 39-60. 